\title{
Intracytoplasmic stable expression of IgG1 antibody targeting NS3 helicase inhibits replication of highly efficient hepatitis C Virus 2a clone
}

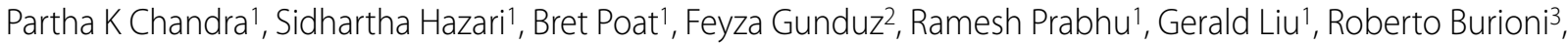 \\ Massimo Clementi 3 , Robert F Garry ${ }^{4}$ and Srikanta Dash ${ }^{* 1,2}$
}

\begin{abstract}
Background: Hepatitis C virus (HCV) infection is a major public health problem with more than 170 million cases of chronic infections worldwide. There is no protective vaccine currently available for HCV, therefore the development of novel strategy to prevent chronic infection is important. We reported earlier that a recombinant human antibody clone blocks viral NS3 helicase activity and inhibits replication of HCV $1 \mathrm{~b}$ virus. This study was performed further to explore the mechanism of action of this recombinant antibody and to determine whether or not this antibody inhibits replication and infectivity of a highly efficient JFH1 HCV 2a virus clone.

Results: The antiviral effect of intracellular expressed antibody against the HCV 2a virus strain was examined using a full-length green fluorescence protein (GFP) labeled infectious cell culture system. For this purpose, a Huh-7.5 cell line stably expressing the NS3 helicase gene specific lgG1 antibody was prepared. Replication of full-length HCV-GFP chimera RNA and negative-strand RNA was strongly inhibited in Huh-7.5 cells stably expressing NS3 antibody but not in the cells expressing an unrelated control antibody. Huh-7.5 cells stably expressing NS3 helicase antibody effectively suppressed infectious virus production after natural infection and the level of HCV in the cell free supernatant remained undetectable after first passage. In contrast, Huh-7.5 cells stably expressing an control antibody against influenza virus had no effect on virus production and high-levels of infectious HCV were detected in culture supernatants over four rounds of infectivity assay. A recombinant adenovirus based expression system was used to demonstrate that Huh-7.5 replicon cell line expressing the intracellular antibody strongly inhibited the replication of HCV-GFP RNA.
\end{abstract}

Conclusion: Recombinant human anti-HCV NS3 antibody clone inhibits replication of HCV 2a virus and infectious virus production. Intracellular expression of this recombinant antibody offers a potential antiviral strategy to inhibit intracellular HCV replication and production.

\section{Background}

Hepatitis $\mathrm{C}$ virus ( $\mathrm{HCV}$ ) infection is a blood borne infectious disease that affects the liver. Only a small fraction of infected individuals clear the HCV infection naturally. In the majority of cases, the virus infection overcomes the host innate and adaptive immune responses leading to a stage of chronic infection. It has been well recognized that chronic $\mathrm{HCV}$ infection often leads to a progressive liver disease including cirrhosis and liver cancer. There

\footnotetext{
* Correspondence: sdash@tulane.edu

1 Department of Pathology and Laboratory Medicine, Tulane University Health Sciences Center, 1430 Tulane Avenue, New Orleans, LA-70112, USA

Full list of author information is available at the end of the article
}

are 170 million people representing 3\% of the world's population that are chronically infected with $\mathrm{HCV}$. The incidence of new infection continues to rise each year at the rate of 3-4 million [1]. Therefore, HCV infection is considered a major health-care problem worldwide. At present there is no prophylactic antibody or therapeutic vaccine available. The only treatment option for chronic $\mathrm{HCV}$ infection is the combination of interferon and ribavirin [2]. This therapy is not effective in clearing all chronic HCV infections. Interferon therapy is also very costly and has substantial side effects. There is a need for 
the development of improved antiviral therapies for the treatment of chronic $\mathrm{HCV}$ infection.

Hepatitis $\mathrm{C}$ virus is a positive-stranded RNA virus containing a single RNA genome of 9600 nucleotides in length [3]. The virus genome contains a short 341 nucleotides untranslated region (5'UTR) followed by a long open reading frame (ORF), ending with a short $3^{\prime}$ untranslated region. The HCV genome can persist in the infected liver cells due to continuous replication of positive-stranded RNA genome. The 5' UTR of HCV RNA is crucial for the initiation of protein synthesis. This component of viral genome recognizes the host ribosome and translates HCV proteins by an IRES dependent mechanism. A single large polyprotein of 3010 amino acids is translated from the long open reading frame (ORF) encoded within the viral RNA genome. This large protein is then cleaved into 10 different individual proteins by the combined action of the cellular and viral proteases. The viral core, E1, E2, and P-7 proteins are called the structural proteins required for the production of infectious virus particles, their secretion and infection. The remaining non-structural proteins (NS2, NS3, NS4A, NS4B, NS5A, NS5B) are essential for replication of HCV positive and negative strand RNA. Among these non-structural proteins, NS3 is the viral protease and NS5B is the viral polymerase. These two proteins have been the targets of novel drug discovery $[4,5]$. There are now large numbers of $\mathrm{HCV}$ inhibitors in the clinical developments targeting these two proteins and these new drugs in combination may improve the treatment of chronic $\mathrm{HCV}$ infection [6].

Several novel antiviral strategies also have been developed using HCV cell culture models including antisense oligonucleotides [7-10], siRNAs [11-15], and recombinant antibodies [16-34]. Hepatitis $C$ virus shows chronic persistent infection in the liver, even in the presence of circulating antibodies to both the structural and nonstructural proteins. The vast majority of these circulating antibodies do not inhibit intracellular virus production and replication. Antibody-mediated neutralization of intracellular and extracellular virus replication and infection is a novel approach to treat chronic viral infection. The rationale of the current study is to develop an intracellular treatment approach for chronic HCV infection by using recombinant antibody technology. During the past few years, significant progress has been made in the design, selection, and production of engineered antibodies $[35,36]$. Antibodies can be reduced in size, rebuilt into multivalent molecules and conjugated with drugs, toxins, or radioisotopes for the treatment of cancer, autoimmune disorders, graft rejection, and infectious diseases.

We have developed a human monoclonal antibody clone derived from a chronically infected patient that effectively inhibits NS3 helicase activity [29-31]. We showed that intracellular expression of this recombinant antibody clone from a plasmid vector inhibits helicase activity and replication of $\mathrm{HCV} 1 \mathrm{~b}$ virus [30]. Here we further explore the mechanism of action of this recombinant antibody and demonstrate that it also inhibits replication of another HCV strain. A Huh-7.5 cell expressing NS3 antibody was developed. We show that replication and infectivity of HCV 2a virus was effectively inhibited in Huh-7.5 cells expressing NS3 antibody. The replication and infectivity of HCV 2a virus was not altered in Huh7.5 cells expressing control antibody against influenza virus.

\section{Materials and methods \\ Cell lines and plasmid clones}

Huh-7.5 cells were obtained from the laboratory of Dr. Charles M Rice (Center for the Study of Hepatitis C, The Rockefeller University, New York) and cells were cultured in Dulbecco's Modified Eagle Medium (DMEM; Invitrogen, San Diego, CA) with high glucose supplemented with non-essential amino acids, sodium pyruvate and 5\% fetal bovine serum. A full-length JFH-1 clone and replicon plasmid (pSGR-JFH1) were obtained from Dr. Takaji Wakita [37] (National Institute of Infectious Diseases, Tokyo, Japan). Full-length and sub-genomic JFH1-GFP was constructed by inserting the coding sequence of green fluorescence protein in the NS5A gene described before [38].

\section{Development of stable Huh-7.5 cell lines expressing $\lg G 1$ antibody}

The construction of plasmid vector pFab-CMV-NS3 $(\mathrm{L}+\mathrm{H})$ containing light chain, heavy chain, $\mathrm{CH} 2-\mathrm{CH} 3$, and part of the hinge region for a germline immunoglobulin 1 was described previously [30]. This vector allows conversion of recombinant Fab antibody into a complete IgG1 antibody in the transfected cells. Huh-7.5 cells were electroporated with $10 \mu \mathrm{g}$ of pFab-CMV $(\mathrm{H}+\mathrm{L})$ plasmid DNA. After 24 hours, cells were selected with a growth medium containing G-418 $(500 \mu \mathrm{g} / \mathrm{ml})$. A control stable Huh-7.5 cell line was prepared that expressed IgG1 antibody targeted to influenza A viruses (clone Fab-9) [39]. These two Huh7.5 lines stably expressing intracellular antibody were cultured in a growth medium containing G-418 $(500 \mu \mathrm{g} / \mathrm{ml})$. Intracellular expression of IgG1 antibody in these two stable cell lines was confirmed by immunofluorescence microscopy. Briefly, cells cultured in chamber slides were washed with phosphate-buffered saline (PBS) pH 7.4 twice, air-dried and fixed with chilled acetone for $5 \mathrm{~min}$. The cells were permeabilized by the treatment with $0.05 \%$ saponin for $10 \mathrm{~min}$ at room temperature. Blocking was performed with $5 \%$ fetal bovine serum (FBS) diluted in a minimum essential medium for $5 \mathrm{~min}$ at room temperature. The slides were washed with 
PBS thrice for $5 \mathrm{~min}$ each. The cells were incubated with goat anti-human phycoerythrin conjugated antibody (anti-human-IgG- $\gamma$ chain specific-R-Phycoerythrin, Sigma-Aldrich, Saint Louis, MO) at 1:50 dilution (in DMEM+5\% FBS) for 1 hour. When staining was completed, the slides were washed three times with PBS and mounted with hoechst dye (H33342, Calbiochem, Darmstadt, Germany) at a concentration of $10 \mu \mathrm{g} / \mathrm{ml}$ prepared in water containing $50 \%$ glycerol. Finally, the slides were examined under a fluorescence microscope at $563 \mathrm{~nm}$ for the red fluorescence and $340 \mathrm{~nm}$ for blue fluorescence. For each area, two sets of pictures were generated. Superimposing blue with red fluorescence using the Abode Photoshop computer software (V 7.0) generated the final image.

\section{Replication Assay}

The effect of an intracellular antibody expression in Huh7.5 cells on the replication of full-length JFH1-GFP RNA genome was examined by using a transfection based replication assay. Full-length in vitro HCV-GFP RNA transcripts were prepared from XbaI digested linearized pJFH1-GFP plasmid by using a commercially available MEGA script kit (Ambion Inc, Austin, TX). The HCV RNA pellet was re-suspended in nuclease free water and $20 \mu \mathrm{g}$ aliquots of this RNA was stored at $-80^{\circ} \mathrm{C}$. Approximately, $2 \times 10^{7}$ cells were re-suspended in $400 \mu \mathrm{l}$ of serum free DMEM, mixed with $20 \mu \mathrm{g}$ of in vitro transcribed RNA and was electroporated using a Gene Pulser Xcell apparatus (Bio-Rad Laboratories Inc. Hercules, CA) with the condition $260 \mathrm{~V}, 960 \mu \mathrm{F}$. Following this step, cells were cultured in DMEM with 10\% fetal bovine serum. The expression of GFP due to HCV replication was monitored under a fluorescence microscope (Olympus $1 \times 70$ ) with every 24 hours interval and the images were captured using an Olympus DP-71 digital camera. Positiveand negative-strand HCV-RNA in the transfected cells was detected by ribonuclease protection assay (RPA). Briefly, total RNA was isolated from the HCV transfected cells every 24 hours by the GITC method and subjected to RPA using a probe targeted to the 5'UTR of HCV. The same amounts of the RNA extracts were subjected to RPA for GAPDH mRNA. To detect HCV RNA, we prepared a plasmid construct called pCR-II-NT-218, which have the sequence of 79-297 nucleotides of 5'UTR sequence of the JFH-1 clone. This plasmid was linearized with HindIII enzyme and positive strand RNA probe was prepared using T7 RNA polymerase to detect the HCV negative strand RNA. Likewise, this plasmid was linearized with the $\mathrm{Xba} I$ restriction enzyme and Sp6 RNA polymerase was used to prepare a negative-strand RNA probe for the detection of a positive-strand HCV RNA. We used a linearized plasmid pTRI-GAPDH-human antisense control template was used to prepare probe to detect GAPDH mRNA using Sp6 RNA polymerase (Ambion Inc., Austin, TX).

\section{Infectivity Assay}

The effect of intracellular antibody expression on production of infectious $\mathrm{HCV}$ was examined by multicycle infectivity assay [38]. Huh- 7.5 cells were transfected with $20 \mu \mathrm{g}$ of in vitro transcribed full-length JFH1-GFP RNA by electroporation method. After 96 hours, cells were collected by scraping and lysed by four rounds of freezethaw cycles. The cell lysates were clarified by centrifugation at $3400 \mathrm{rpm}$ for five minutes. The clear supernatant was collected and titer of $\mathrm{HCV}$ in the supernatant was determined by real-time RT-PCR using a primer set targeted to the 5'UTR. Tissue culture infective dose (TCID50 and MOI) of the virus stock was determined using 10-fold serial dilution of the virus containing supernatant using 2-well Lab-Tek chamber slides (Nalge-Nunc International, Rochester, New York). Huh-7.5 cells stably expressing intracellular antibody were seeded at a density of $1 \times 10^{6}$ cells $/ 100 \mathrm{~mm}$ plate. The next day the culture medium was removed and cells were infected with $3 \mathrm{ml}$ of culture supernatant containing infectious virus $(5 \times$ $10^{5}$ virus particles/ml, MOI 1.5). After overnight incubation, the cells washed three times using $10 \mathrm{ml}$ of PBS and incubated with $10 \mathrm{ml}$ of complete growth medium. Cell free culture supernatants were collected after 96 hours, clarified by centrifugation. Three $\mathrm{ml}$ of culture supernatants was used to infect new batch of antibody expressing Huh-7.5 cells. The infectivity assay was performed up to four cycles each using the identical procedure. At the end of infectivity assay, RNA was isolated from $1 \mathrm{ml}$ of culture supernatants and HCV RNA titer was measured by realtime RT-PCR.

\section{Real time reverse transcription polymerase chain reaction (RT-PCR)}

Real time RT-PCR was performed to quantify HCV RNA levels in the infected cell culture using a published protocol [40]. The 243 bp HCV DNA was amplified from the RNA extract by reverse transcription polymerase chain reaction using the outer sense (OS) primer 5'-GCAGAAAGCGCCTAGCCATGGCGT-3' (67-90) and outer anti-sense (OAS) primer 5'-CTCGCAAGCGCCCTATCAGGCAGT-3' (287-310). First the complementary DNA synthesis was performed from positive-strand HCV-RNA using an outer anti-sense primer (OAS) targeted to the highly conserved 5'UTR region of HCV in 20 $\mu \mathrm{l}$ volume. Briefly, $2 \mu \mathrm{g}$ of total cellular RNA were mixed with $1 \mu \mathrm{l}$ OAS primer $(200 \mathrm{ng} / \mu \mathrm{l})$, denaturized at $65^{\circ} \mathrm{C}$ for 10 minutes and annealed at room temperature. Avian myeloblastosis virus (AMV) reverse transcriptase (10 U) (Promega, Madison, WI) was added and incubated at $42^{\circ} \mathrm{C}$ for 60 minutes in the presence of $50 \mathrm{mmol} / \mathrm{L}$ Tris, 
$\mathrm{pH}$ 8.3, $50 \mathrm{mmol} / \mathrm{L}$ ethylenediaminetetraacetic acid (EDTA), $500 \mathrm{nmol} / \mathrm{L} \mathrm{dNTP,} 250 \mathrm{nmol} / \mathrm{L}$ spermidine, and 40 U RNasin (Promega, Madison, WI). The cDNA was stored at $-20^{\circ} \mathrm{C}$ until use. SYBR Green real time PCR amplification was performed in $20 \mu \mathrm{l}$ of volume containing $10 \mu \mathrm{l}$ of SYBR Green ER qPCR SuperMix, $1 \mu \mathrm{l}(250$ $\mathrm{ng} / \mathrm{ul}$ ) of sense and antisense primer with $4 \mu \mathrm{l}$ of cDNA and $4 \mu \mathrm{l}$ of distilled water. All samples were run in triplicate. The amplification was carried out using the standard program recommended by Bio-Rad Laboratory that includes: $50^{\circ} \mathrm{C}$ for 2 minutes, $95^{\circ} \mathrm{C}$ for 8 minutes, then additional 50 cycles wherein each cycle consists of a denaturation step at $95^{\circ} \mathrm{C}$ for 10 seconds, and annealing and extension step at $60^{\circ} \mathrm{C}$ for 30 seconds. At the end of the amplification cycles, melting temperature analysis was performed by a slow increase in temperature $\left(0.1^{\circ} \mathrm{C} /\right.$ s) up to $95^{\circ} \mathrm{C}$. Amplification, data acquisition, and analysis were performed on CFX96 Real-Time instrument using CFX manager software (Bio-Rad, Hercules, CA).

\section{Construction of adenovirus vector containing $\lg \mathrm{G} 1$ antibody}

A replication defective adenovirus construct carrying the gene for the recombinant human antibody (Ad-IgG1) was prepared using standard PCR and cloning methods. We used the pFab-CMV-NS3 $(\mathrm{L}+\mathrm{H})$ plasmid construct to prepare a recombinant adenovirus vector (30). The cloning process was carried out in multiple steps. First, the heavy chain antibody expression cassette containing the CMV promoter-antibody ORF, Fd termination sequence and heavy chain polyadenylation sequences were PCR amplified and assembled in pGEM-7Z(f+) vector (Promega, Madison, WI) using XhoI and BamHI sites. An unique HindIII site was introduced before the $\mathrm{XhoI}$ and BamHI site such that the entire antibody expression cassette can be excised from the pGEM-7Z $\left(f_{+}\right)$plasmid and inserted into the adenovirus pShuttle Vector (QBIOgene, AES1020, without CMV promoter) using a unique HindIII site. After cloning, the exact orientation of the heavy chain antibody expression cassette was confirmed by DNA sequence analysis. At the second step, the antibody light chain gene was inserted into the same plasmid shuttle (pShuttle vector without CMV promoter) using two PCR fragments. The first light chain CMV promoterleader sequence and light chain ORF was cloned into a pShuttle plasmid using Kpn1 and XbaI sites. Then the remaining light chain termination sequence and polyadenylation sequences were introduced into the same pShuttle vector using $\mathrm{XbaI}$ sites. The orientation of the CMV promoter of the light chain and heavy chain reading frame in the resulting plasmid was confirmed by restriction analysis. The recombinant plasmid is called pShuttle CMV NS3 $(\mathrm{H}+\mathrm{L})$. The antiviral effect of recombinant antibody clone was confirmed again by transfection into a replicon cell line. The pShuttle CMV NS3 $(\mathrm{H}+\mathrm{L})$ antibody gene was then transferred into the adenoviral genome (pAdEasy, QBiogene, Carlsbad, CA) by homologous recombination. The recombinant adenovirus plasmid was examined by restriction digestion analysis. The recombinant adenovirus plasmid was linearized with a PacI restriction enzyme and transfected to QBI-293 cells (QBiogene, Carlsbad, CA). After several weeks, the recombinant adenovirus was plaque purified and amplified on 293 cells. Large-scale purification of the recombinant adenovirus was performed by $\mathrm{CsCL}$ gradient centrifugation. The titer of recombinant adenovirus (virus particles $/ \mathrm{ml}$ ) was determined by using absorbance at optical density at $260 \mathrm{~nm}$, a standard protocol supplied in the kit. A recombinant adenovirus carrying firefly luciferase (Ad-Luc) was used as a control to exclude the non-specific effect of recombinant adenovirus infection on $\mathrm{HCV}$ replication.

\section{Immunocytochemistry}

Huh-7.5 cells were cultured in chamber slides and after 24 hours they were infected with a different concentration of recombinant adenovirus (Ad-IgG1). The intracellular expression of a recombinant antibody was examined by an immunostaining method using goat anti-human antibody (Sigma-Aldrich, Saint Louis, MO). Briefly, Huh7.5 cells cultured in chamber slides were washed with phosphate-buffered saline (PBS) pH 7.4 twice, air-dried and fixed with chilled acetone for five minutes. The cells were permeabilized by the treatment with $0.05 \%$ saponin for 10 minutes at room temperature. Blocking was performed with $5 \%$ normal goat serum diluted in a minimum essential medium for 30 minutes at room temperature. Blocking for endogenous biotin-avidin was performed using an avidin/biotin blocking kit (Vector Laboratories Inc., Burlingame, $\mathrm{CA}$ ). Blocking for endogenous peroxidase was carried out with $0.9 \% \mathrm{H}_{2} \mathrm{O}_{2}$ for 30 minutes at room temperature. The cells were incubated with a biotin conjugated goat anti-human IgG antibody (1:500 dilution, Sigma-Aldrich, Saint Louis, MO). The slide was then washed three times and incubated with an anti-mouse biotin conjugated antibody (1:1000) for one hour at room temperature. The slides were then washed and incubated for 30 minutes with Elite avidin-biotin peroxidase complex (Vector Laboratories Inc., Burlingame, CA). The slides were reacted with diaminobenzidine for $10 \mathrm{~min}$ utes. Counterstaining was performed with hematoxylin for one minute. After dehydration, the slides were mounted with per mount and observed by light microscopy.

\section{Western blotting}

To make sure that the intracellular expressed antibody molecule processed accurately, western blot analysis was 
performed using lysates prepared from the adenovirus infected Huh-7.5 cells. Immunoblotting was performed with a peroxidase conjugated rabbit anti-human antibody at a dilution of 1:500. (Sigma-Aldrich, Saint Louis, MO). The membrane was developed using the enhancedchemiluminescence detection kit (ECL kit) (Amersham Pharmacia, Piscataway, NJ) and was exposed to chemiluminescent-sensitive film (Kodak Rochestor, NY).

\section{Results}

Huh-7.5 cells stably expressing NS3 antibody inhibits HCV 2 a replication

The initial step of this study was the development of a Huh-7.5 cell line with stable expression of intracellular antibody. The recombinant human antibody gene was introduced in to a mammalian plasmid expression vector that expresses the heavy and light chain antibody gene using the two identical CMV promoters (Fig. 1). This allows production of equal amounts of heavy and light chains of the antibody molecule in the cytoplasm. A stable Huh-7.5 cell line expressing NS3 antibody targeting the HCV NS3 helicase was prepared. Another Huh-7.5 cell line stably expressing antibody against influenza virus was used as a control. We show that both the Huh-7.5 cell lines expresses IgG1 antibody in the cytoplasm by immunological staining (Fig. 2). Stable intracellular antibody expression ensured no cellular toxicity since these cells can be propagated in culture for prolonged time. To study the effect of the intracellular antibody on full-length HCV RNA replication, we used a chimeric full-length JFH1-GFP clone where the coding sequence of GFP was introduced in the NS5A region [38]. We have shown that this clone replicates in Huh-7.5 cells and produces positive- and negative-strand HCV RNA by ribonuclease protection assay. To determine the antiviral effect of intracellular antibody expression on the replication of full-length JFH-1 clone, full-length JFH1-GFP HCV-RNA was transfected to stable Huh-7.5 cell lines expressing an intracellular antibody. The effect of intracellular antibody expression on replication of $\mathrm{HCV}$ was determined by RPA assay at 0, 24, 48, 72 and 96 hours. The results of RPA assay for the detection of positive- and negative-

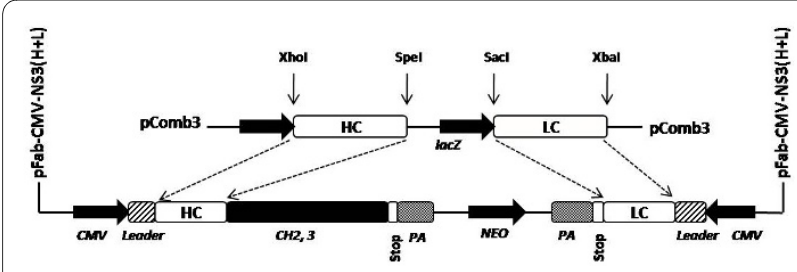

Figure 1 The schematic diagram of the recombinant antibody construct. The recombinant Fab antibody clone was expressed in mammalian cells using the expression vector (pFab-CMV-NS3 $(\mathrm{H}+\mathrm{L})$ that has two identical CMV promoters. This vector was used to generate stable Huh-7.5 cells expressing intracellular lgG1 antibody.

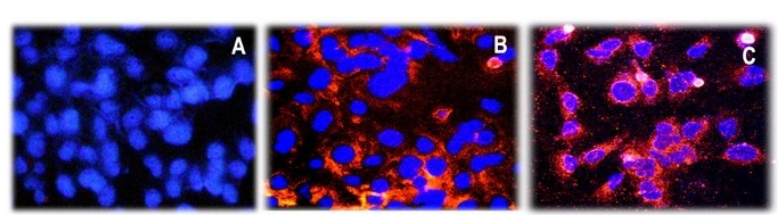

Figure 2 Immunofluorescence staining showing stable intracytoplasmic expression of NS3 helicase specific antibody and control antibody in Huh-7.5 cells. Stable Huh-7.5 cells were seeded in 2-well chamber slides and after 48 hours cells were stained with phycoerythrin conjugated anti-human IgG1 antibody at a dilution of 1:50. After this step, cells were counterstained with hoechst dye. A: Negative staining of control Huh 7.5 cells. B: Huh 7.5 cells stably expressing a control antibody. C: Huh 7.5 cells stably expressing NS3 antibody.

strand RNA detection are shown in Fig. 3. The levels of positive-strand RNA decreased over time and remained undetectable at 96 hours only in the cells expressing NS3 antibody. In contrast, positive-strand HCV RNA was detected at all time points in cells expressing an unrelated control antibody or Huh-7.5 cells without antibody expression. HCV is a positive-strand RNA virus that replicates by a negative-strand RNA intermediate. The effect of antibody expression on the replication of JFH1-GFP RNA in Huh-7.5 cells was examined by measuring HCV negative-strand RNA levels by RPA. HCV negativestrand RNA remained undetected in Huh-7.5 cells expressing NS3 antibody. In contrast, HCV negativestrand RNA was detected in Huh-7.5 cells expressing control antibody and Huh-7.5 cells without antibody expression. These results suggest that HCV replication was terminated in cells stably expressing intracellular helicase antibody, while in control cells HCV replication was unaltered. The antiviral effect of intracellular helicase antibody was examined by green fluorescence protein expression in a kinetic study. The expression of HCVGFP chimera protein specifically decreased in cells expressing NS3 antibody and the protein expression remained undetectable at 96 hours (Fig 4). The level of viral RNA in the antibody expressing cells and control Huh-7.5 cells after transfection was quantitated by using real-time RT-PCR using a primer set targeted to the highly conserved 5'UTR region. HCV RNA levels progressively decreased in Huh-7.5 cells expressing NS3 antibody (Fig. 5). There are significant differences in the levels of HCV RNA between the NS3 antibody expressing cells and two control cell lines. Taken together these results indicate that full-length JFH1-GFP RNA replication was selectively inhibited in Huh-7.5 cells expressing intracellular NS3 antibody.

\section{Huh-7.5 cells stably expressing NS3 antibody inhibits production of intracellular and extracellular virus}

The effect of intracellular NS3 antibody on the production of infectious virus particles was examined by per- 


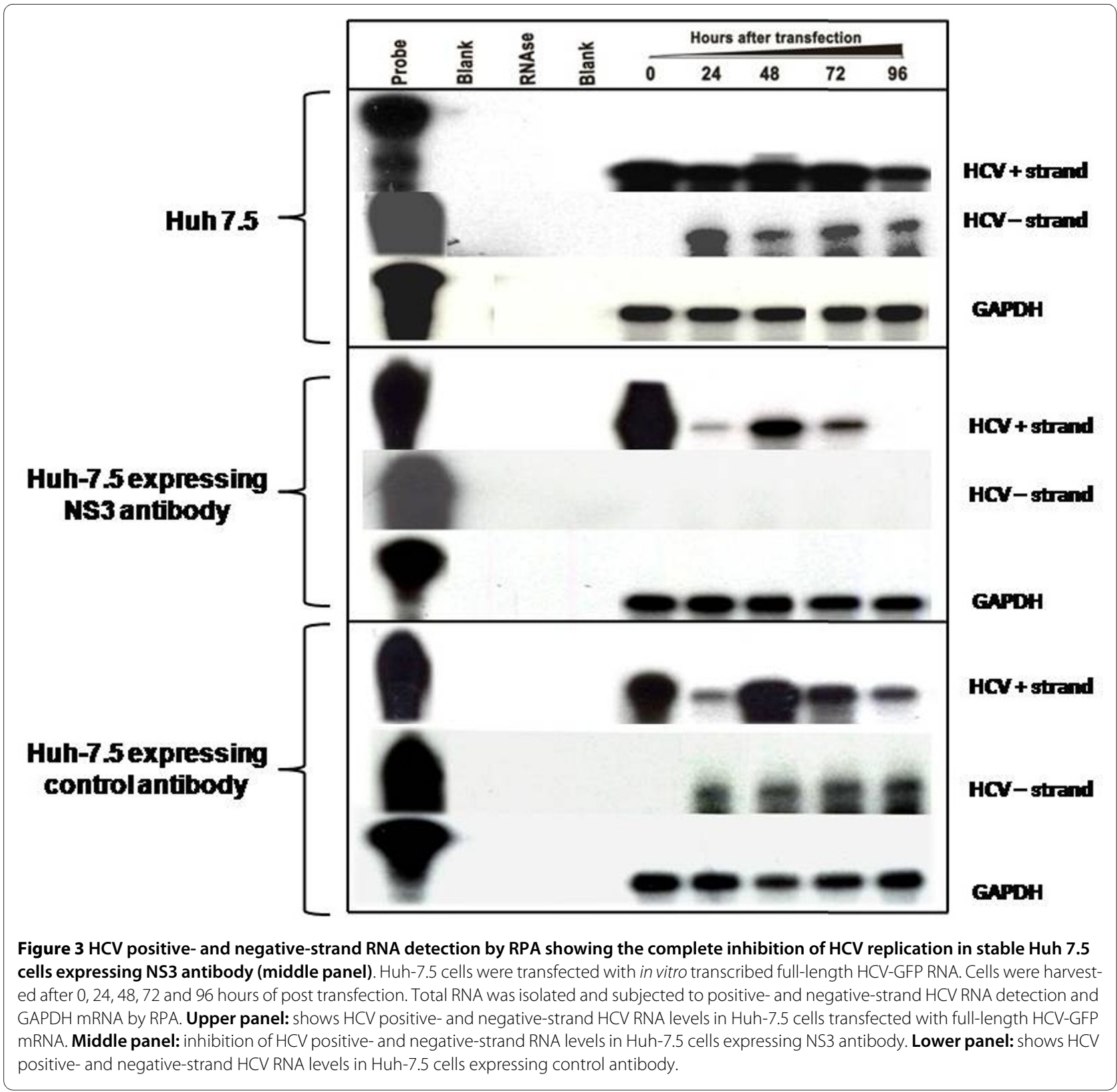

forming multicycle infectivity assay. We previously established infectivity assay for HCV using the JFH1-GFP clone. The infectivity of $\mathrm{HCV}$ can be directly determined by examining the expression of GFP under a fluorescence microscope. Huh-7.5 cells expressing NS3 antibody and Huh-7.5 cells expressing control antibody were infected with infectious HCV and replication of HCV-GFP chimera after natural infection was examined in a kinetic study. We found that the replication of HCV-GFP chimera virus was inhibited in the Huh-7.5 cells expressing NS3 antibody since we did not see any GFP expression (Fig. 6). In contrast, HCV-GFP expression in the infected cells was detected in both the control Huh-7.5 cells. The infectivity assay was serially repeated an additional three cycles using $3 \mathrm{ml}$ of culture supernatants (MOI 1.5). The production of infectious $\mathrm{HCV}$ in Huh-7.5 cells with or without antibody expression was measured by real-time RT-PCR. The results of these experiments (Fig. 7) indicate that there is a significant drop in the HCV RNA levels after first passage in Huh-7.5 cells expressing NS3 antibody. The levels of HCV RNA in the supernatants did not change in Huh-7.5 cells and Huh-7.5 cells expressing control antibody. These results clearly indicate that the NS3 antibody prevents intracellular HCV RNA replication leading to reduced virus particle formation and secretion. 

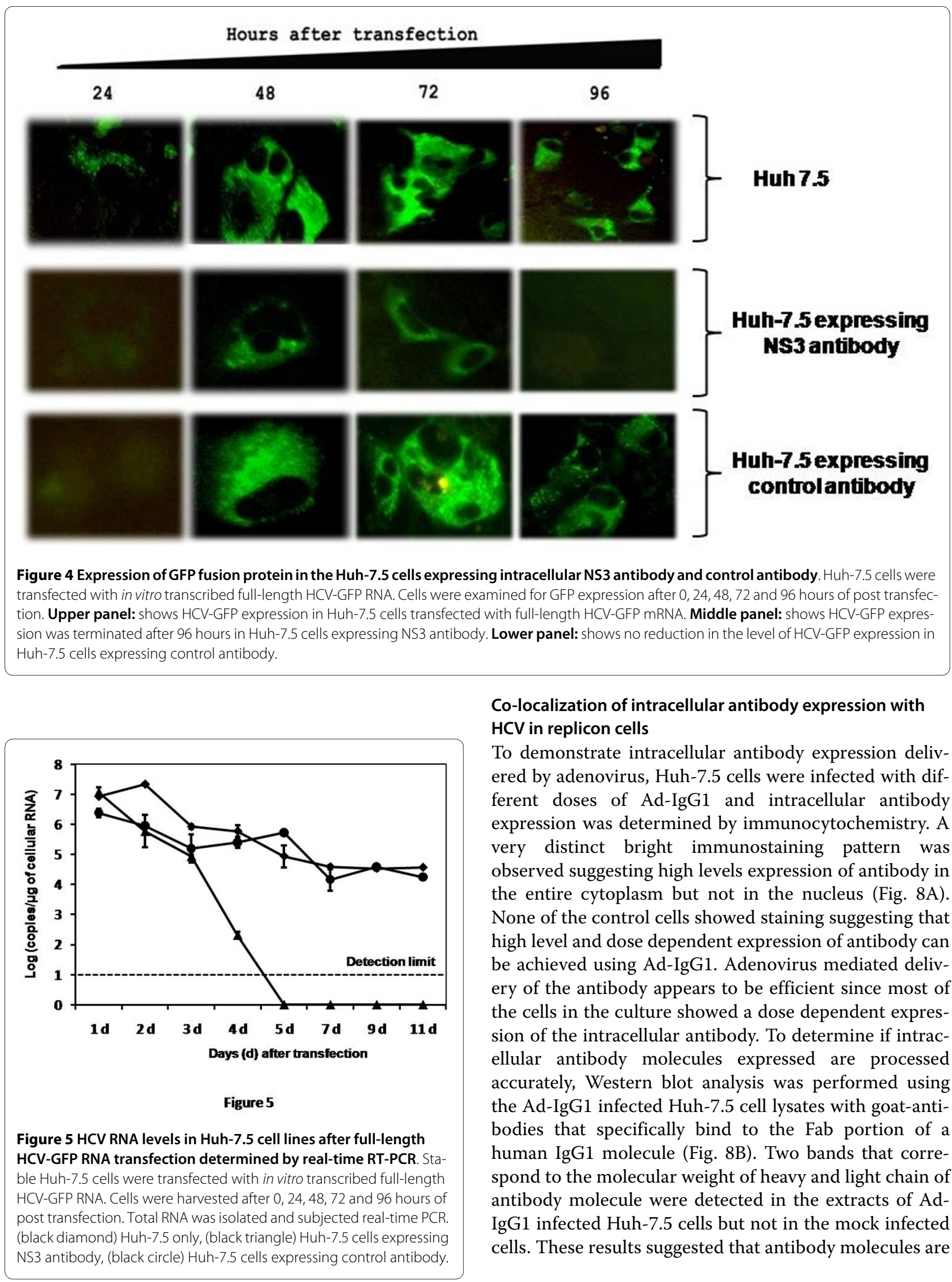

Co-localization of intracellular antibody expression with $\mathrm{HCV}$ in replicon cells

To demonstrate intracellular antibody expression delivered by adenovirus, Huh-7.5 cells were infected with different doses of Ad-IgG1 and intracellular antibody expression was determined by immunocytochemistry. A very distinct bright immunostaining pattern was observed suggesting high levels expression of antibody in the entire cytoplasm but not in the nucleus (Fig. 8A). None of the control cells showed staining suggesting that high level and dose dependent expression of antibody can be achieved using Ad-IgG1. Adenovirus mediated delivery of the antibody appears to be efficient since most of the cells in the culture showed a dose dependent expression of the intracellular antibody. To determine if intracellular antibody molecules expressed are processed accurately, Western blot analysis was performed using the Ad-IgG1 infected Huh-7.5 cell lysates with goat-antibodies that specifically bind to the Fab portion of a human IgG1 molecule (Fig. 8B). Two bands that correspond to the molecular weight of heavy and light chain of antibody molecule were detected in the extracts of AdIgG1 infected Huh-7.5 cells but not in the mock infected cells. These results suggested that antibody molecules are 


\section{Hours after infection}

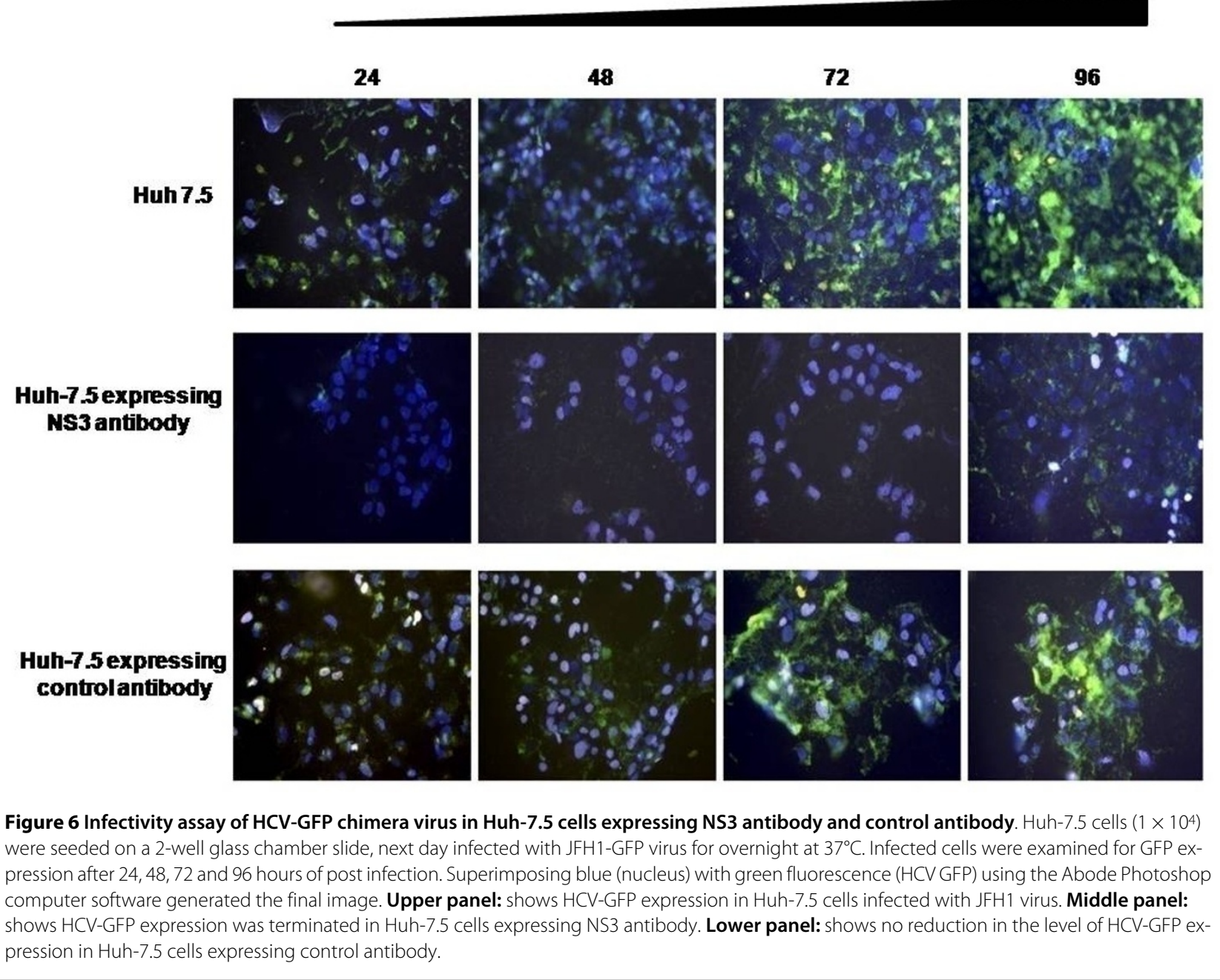

accurately processed and expressed high levels in the cytoplasm of a liver derived cell line. In order to determine the extent of inhibition of $\mathrm{HCV}$ replication that occurred due to intracellular expression of the antibody in the cytoplasm of individual replicon cells, we performed co-localization experiments using a GFP-tagged replicon cell line. This cell line supports high levels of replication of HCV JFH1-sub genomic RNA that can be directly visualized by GFP expression. This S-3/GFP replicon cell was infected with Ad-IgG1 virus. After 72 hours, the antiviral efficacy of intracellular antibody expressed from recombinant adenovirus vector was examined by flow cytometric analysis (Fig. 9A). The S-3/GFP replicon cells expressed high level of GFP that can be detected by flow analysis. The expression of GFP was not altered when infected with Adv-Luciferase. The GFP expression was inhibited in S-3/GFP replicon cells infected with Adenovirus carrying NS3 antibody gene. We then performed a co-localization experiment using the S-3/GFP cells where intracellular antibody expression was examined using a phycoerythrin-labeled goat-anti-human IgG1 antibody and HCV was detected by GFP expression. Slides were randomly examined for antibody expression as well as GFP expression under a fluorescence microscope and fluorescence pictures in the same areas were composed. We anticipate that if the antibody expression inhibits $\mathrm{HCV}$ replication then one would expect not to see GFP expression in the same replicon cells that express intracellular antibody (Fig. 9B-F), suggesting that cells expressing NS3 antibody showed negative expression for GFP. Likewise cells showing GFP expression did not show antibody expression. We did not observe hepatocytes showing positive expression of both HCV (GFP) as well as intracellular antibody (red) in the entire culture. These results suggest that intracellular antibody expression in individual replicon cells resulted in the inhibition of GFP expression. 


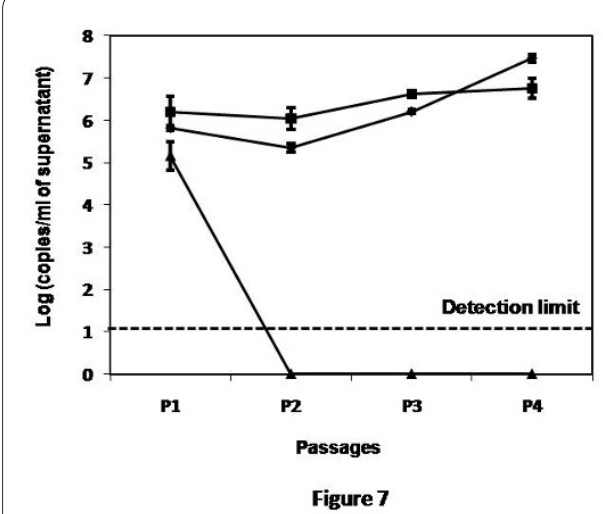

Figure 7 The production of infectious HCV particles in the Huh7.5 cells expressing NS3 antibody and control antibody. $1 \times 10^{6}$ cells were seeded in a 100-mm plate was infected with HCV-GFP virus at a $\mathrm{MOI}$ of 1.5. Cell free supernatants were collected every 96 hours. Three milliliter of culture supernatants was used to infect freshly cultured Huh-7.5 cells. The infectivity assay was repeated for four passages. The production of infectious HCV using RNA extracts from $1 \mathrm{ml}$ of culture supernatants was determined by real-time RT-PCR. (black diamond) Huh-7.5 only, (black triangle) Huh-7.5 cells expressing NS3 antibody, (black square) Huh-7.5 cells expressing control antibody.

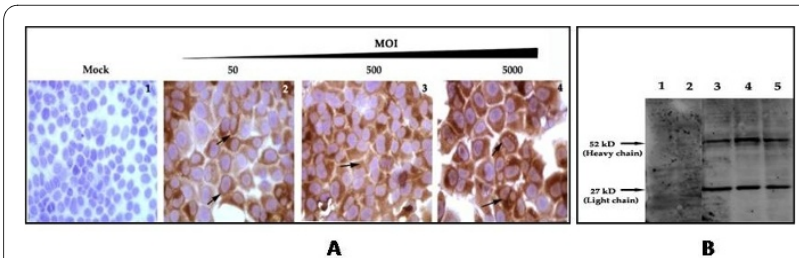

Figure 8 Intracytoplasmic expression of IgG1 antibody delivered by adenovirus vector. Left panel shows dose-dependent intracytoplasmic expression of IgG1 antibody in Huh7.5 cells delivered by adenovirus vector. Huh-7.5 cells were infected with increasing concentrations of Ad-lgG. After 24 hours, intracellular antibody expression was examined by immunostaining with a biotin conjugated goat anti-human IgG1 antibody. Slides were then counterstained with hematoxylin. 1: Mock infected cells showing negative staining. 2-4: Dose dependent intracytoplasmic brown staining was seen in majority of Huh-7.5 cells infected with Ad-lgG virus (A). Western blot analysis showing the presence of light chain and heavy chain antibody in the lysates of Huh-7.5 cells infected with Ad-lgG (B). Huh-7.5 cells were infected with three different batches of recombinant Ad-lgG at a concentration of $4000 \mathrm{MOl}$. Cell lysates were made after 24 hours. $50 \mathrm{\mu g}$ of protein lysates were separated on a 10\% SDS PAGE and transferred to a nylon membrane. Immunoblotting was performed with peroxidase conjugated rabbit anti-human antibody. The membrane was developed using the ECL kit and was exposed to chemiluminescent-sensitive film. 1. Uninfected Huh-7.5 cells. 2 . Huh-7.5 cells infected with an adenovirus luciferase (Ad-Luc). 3-5. Huh-7.5 cells infected with three different batches of plaque-purified virus (Ad-lgG). Two specific bands at molecular weight $27 \mathrm{kD}$ and $52 \mathrm{kD}$ represent the light chain and the heavy chain of the $\lg \mathrm{G} 1$ antibody respectively.

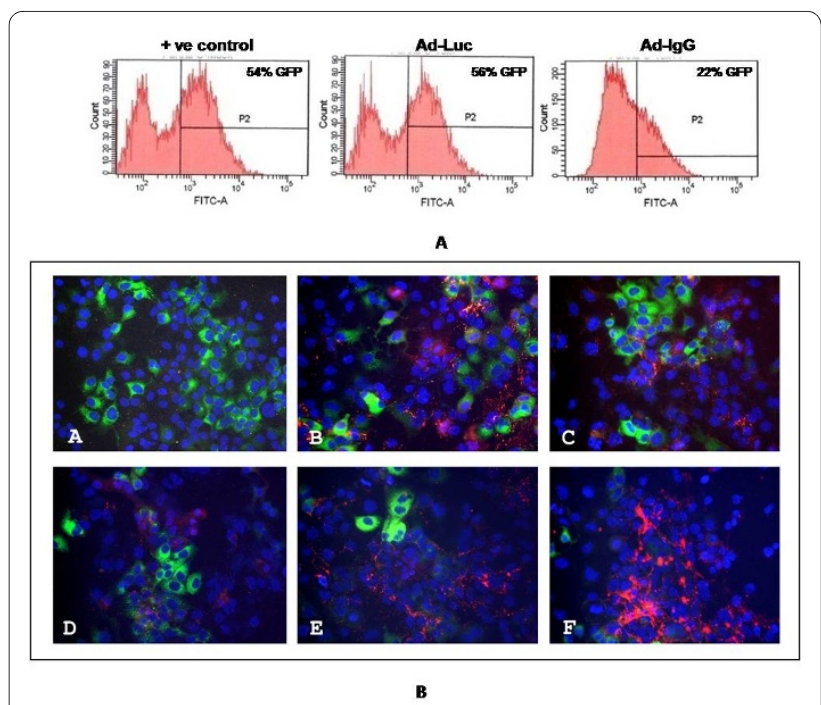

Figure 9 Intracellular antibody expression by adenovirus vector inhibits HCV replication in a sub-genomic GFP based stable replicon cell line. Upper Panel shows flow cytometric analysis of HCVGFP protein level in replicon cell line expressing intracellular NS3 antibody from adenovirus vector. $1 \times 10^{6}$ replicon cells were seeded in a $100 \mathrm{~mm}$ tissue culture dishes and next day they were infected with recombinant adenovirus (Ad-IgG or Ad-Luc). After 72 hours, cells were harvested by using trypsin-EDTA and then analyzed by a flow cytometer where GFP expressing cells were quantified. Lower panel shows that intracellular antibody expression eliminates HCV GFP expression in replicon cell line in a co localization experiment. Replicon cells $(2 \times$ $10^{4}$ ) were grown in a 2-well chamber slide and after 24 hours cells were infected with increasing concentration of Ad-lgG (ranged from 50, 500, 1000, 2000 and $4000 \mathrm{MOI}$ ). After 72 hours, cells were fixed for $15 \mathrm{~min}$ with $2 \%$ paraformaldehyde. This fixation did not destroy GFP expression. Intracellular expression of antibody was performed using phycoerythrin conjugated anti-human IgG1 antibody (1:50 dilution). After this step, cells were washed and counterstained with hoechst dye. Multiple areas in the slide were randomly examined. Three sets of pictures from the same area were taken. First, the intracellular antibody expression (red), second for the GFP expression and third blue nuclear staining of stained cells under a fluorescent microscope. Finally, the combined picture in the same area was composed using Abode Photoshop (version 7.0) computer software. A: Shows fairly high-level expression of HCV GFP the expression in S-3/GFP replicon cells. B-F: Colocalization of GFP and intracellular antibody. The expression of GFP was decreased in cells infected with Ad-IgG and panel (B-E) the co-localization HCV-GFP (green) and intracellular antibody (red).

\section{Discussion}

We described here a novel intracellular treatment approach for chronic HCV infection using a humanized recombinant antibody targeting the viral NS3 helicase enzyme. We hypothesized that intracellular expression of the antibody targeting the viral NS3 helicase should inhibit its enzyme activity and HCV replication. The NS3 helicase enzyme is crucial for the HCV genome replication justifying it as potential target for ant-viral therapy [41-43]. As a proof-of-principle, we have already shown 
that transient transfection of this human antibody clone using a plasmid vector into HCV $1 \mathrm{~b}$ replicon cell lines inhibit HCV helicase activity and HCV RNA replication [30]. The replicon-based model lacks the structural proteins and does not produce infectious virus. Therefore we performed this study to determine the success of inhibiting full-length virus genome replication using this antibody clone.

The antiviral property of this antibody clone was examined using the highly efficient JFH1 clone belonging to HCV 2a virus strain. This clone has now been widely used by a number of investigatons to study the replication of full-length virus genome after natural infection using Huh-7.5 cell culture. For this reason, stable Huh-7.5 cell lines were prepared that express HCV NS3 specific antibody and influenza specific control antibody. Huh-7.5 cells expressing intracellular antibody were cultured using a medium containing G-418 selection. We showed that Huh-7.5 cell expresses a recombinant antibody by immunocytochemical staining. To study the effect of antibody expression on HCV RNA replication, the HCVGFP chimeric clone was transfected to Huh-7.5 cells stably expressing either NS3 helicase antibody or a control recombinant antibody. It was shown that $\mathrm{HCV}$ positivestrand RNA, negative-strand RNA levels and NS5A-GFP fusion protein expression was inhibited in Huh-7.5 cells expressing intracellular antibody. The inhibition of viral HCV RNA replication and protein expression was not seen in control Huh-7.5 cells. The level of viral RNA replication was not altered in two other control cell lines, including those expressing an antibody to influenza virus, suggesting that this effect is specific to NS3 helicase antibody expression.

The inhibition of infectious virus particle production in stable antibody expressing Huh-7.5 cell line was examined using an infectious cell culture system for over four cycles of infectivity assay. We showed that the production of HCV in the NS3 antibody expressed Huh-7.5 cells was strongly inhibited and the titer of $\mathrm{HCV}$ remained below the detection limit after first passage. The infectious HCV particle production in two control Huh-7.5 cells were maintained at a relatively high levels in the range of $10^{7}-10^{5}$ copies of HCV RNA $/ \mathrm{ml}$ in the culture supernatants. The results of multicycle infectivity assay support the notion that intracellular NS3 antibody effectively suppressed the virus replication and infectious virus production. The intracellular antibody expression appears to have effectively suppressed the production of infectious $\mathrm{HCV}$. In this study no evidence of escape mutant HCV was observed over four passages. We showed that most of the cells expressed antibody intracellular and inhibited HCV expression by co-localization studies. We showed that the antibody gene is accurately processed and expressed at high levels using an adenovirus vector.

Recombinant antibody-based antiviral strategies for $\mathrm{HCV}$ have been reported by a number of investigators. Some of the recombinant antibodies are directed against structural proteins [17,18,22,23,26-28,32]. Antibodies targeted against E1 and E2 regions can be utilized to neutralize HCV infection to cells in vitro as well as in vivo. Likewise, it is expected that intracellular expression of antibodies targeting the viral core protein should interfere with formation of infectious virus production and packaging. Some investigators have also developed recombinant antibodies targeting the viral non-structural proteins (NS3 protease, helicase and polymerase) of $\mathrm{HCV}$ [20,24,25,29-31]. These antibodies should be used to effectively block the viral protease, helicase and polymerase activity and thereby inhibit intracellular $\mathrm{HCV}$ replication and infection. One of the major problems in this antiviral strategy is the intracellular delivery of the antibody for therapeutic approaches in human. As a proof of principle we have demonstrated that intracellular inhibition of replication of the highly efficient JFH-1 virus clone can be achieved using an antibody clone targeted to the NS3 helicase. We also showed that the intracellular expression of a recombinant antibody to a liver derived cell line efficiently inhibits JFH-1 virus replication and production. The replication-defective adenovirus expresses high levels of therapeutic antibody in cultured cells. This will allow large-scale antibody purification from mammalian cells in culture. We realize that the use of adenovirus has considerable adverse side effects in humans; therefore, future research will focus on developing safer non-viral delivery methods of targeted delivery antibody to liver cells to inhibit HCV replication.

\section{Competing interests}

The authors declare that they have no competing interests.

\section{Authors' contributions}

PKC performed most of the biochemical experiments, participated in the design of the study and wrote the initial draft of the manuscript. SH, RP and GL contributed to construct the replication defective adenovirus carrying the recombinant antibody gene. BP and FG helped in some biochemical experiments. RB, MC supplied the recombinant antibody clones and helped to prepare the manuscript. RFG and SD supervised, helped to design the study and finally edited the manuscript. All authors read and approved the final manuscript.

\section{Acknowledgements}

We thank to Jeanne Frois for critically reading the manuscript. The authors thank Charlie Rice for providing Huh-7.5 cell line, Takaji Wakita for providing JFH-1 clone, Jay Kolls, Louisiana State University (LSU) for Ad-Luc used in the experiments. This work was supported by funds received from the National Institutes of Health (R56Al64617, R21DK070551, R44Al082778), National Cancer Institute (CA127481, CA129776), Gayer Foundation New York, Louisiana Cancer Research Consortium (LCRC), Tulane Cancer Center, Louisiana Board of Reagents. 


\section{Author Details}

'Department of Pathology and Laboratory Medicine, Tulane University Health Sciences Center, 1430 Tulane Avenue, New Orleans, LA-70112, USA 2Department of Medicine, Gastroenterology, Tulane University Health Sciences Center, 1430 Tulane Avenue, New Orleans, LA-70112, USA, ${ }^{3}$ Facoltà di Medicina e Chirurgia, Università Vita-Salute San Raffaele, Via Olgettina, 60 - DiBit2, 20132 Milano, Italy and ${ }^{4}$ Department of Microbiology and Immunology, Tulane University Health Sciences Center, 1430 Tulane Avenue, New Orleans, LA70112, USA

Received: 7 January 2010 Accepted: 7 June 2010

Published: 7 June 2010

\section{References}

1. World Health Organization (WHO): Hepatitis C, Fact Sheet No.164. Revised October 2000. [http://www.who.int/inf-fs/en/fact164.html].

2. Strader DB, Wright T, Thomas DL, Seeff LB: Diagnosis, management, and treatment of hepatitis C. Hepatology 2004, 39:1147-1171.

3. Reed KE, Rice CM: Overview of hepatitis C virus genome structure, polyprotein processing, and protein properties. Curr Top Microbiol Immunol 2000, 242:55-84.

4. Kwong AD, McNair L, Jacobson I, George S: Recent progress in the development of selected hepatitis C virus NS3.4A protease and NS5B polymerase inhibitors. Curr Opin Pharmacol 2008, 8:522-531.

5. Parfieniuk A, Jaroszewicz J, Flisiak R: Specifically targeted antiviral therapy for hepatitis C virus. World J Gastroenterol 2007, 13:5673-5681.

6. Manns MP, Foster GR, Rockstroh JK, Zeuzem S, Zoulim F, Houghton M: The way forward in HCV treatment-finding the right path. Nat Rev Drug Discov 2007, 6:991-1000

7. Alt M, Renz R, Hofschneider PH, Paumgartner G, Caselmann WH: Specific inhibition of hepatitis $C$ viral gene expression by antisense phosphorothioate oligodeoxynucleotides. Hepatology 1995, 22:707-717.

8. Hanecak R, Brown-Driver V, Fox MC, Azad RF, Furusako S, Nozaki C, Ford C, Sasmor H, Anderson KP: Antisense oligonucleotide inhibition of hepatitis $\mathrm{C}$ virus gene expression in transformed hepatocytes. J Virol 1996, 70:5203-5212.

9. Trepanier JB, Tanner JE, Alfieri C: Oligonucleotide-based therapeutic options against hepatitis C virus infection. Antivir Ther 2006, 11:273-287.

10. Wakita T, Wands JR: Specific inhibition of hepatitis $C$ virus expression by antisense oligdeoxynucleotides. In virto model for selection of target sequence. J Biol Chem 1994, 269:14205-14210.

11. Randall G, Grakoui A, Rice CM: Clearance of replicating hepatitis C virus replicon RNAs in cell culture by small interfering RNAs. Proc Natl Acad Sci USA 2003, 100:235-240.

12. Prabhu R, Vittal P, Yin Q, Flemington E, Garry R, Robichaux WH, Dash S: Small interfering RNA effectively inhibits protein expression and negative strand RNA synthesis from a full-length hepatitis $C$ virus clone. J Med Virol 2005, 76:511-519.

13. Grimm D, Kay MA: Therapeutic short hairpin RNA expression in the liver: viral targets and vectors. Gene Ther 2006, 13:563-575

14. Watanabe T, Umehara T, Kohara M: Therapeutic application of RNA interference for hepatitis C virus. Adv Drug Deliv Rev 2007, 59:1263-1276.

15. Chevalier C, Saulnier A, Benureau Y, Fléchet D, Delgrange D, ColbèreGarapin F, Wychowski C, Martin A: Inhibition of hepatitis $C$ virus infection in cell culture by small interfering RNAs. Mol Ther 2007, 15:1452-1462.

16. Bartosch B, Bukh J, Meunier JC, Granier C, Engle RE, Blackwelder WC, Emerson SU, Cosset FL, Purcell RH: In vitro assay for neutralizing antibody to hepatitis $C$ virus: evidence for broadly conserved neutralizing epitopes. Proc Nat/ Acad Sci USA 2003, 100:14199-14204.

17. Burioni R, Bugli F, Mancini N, Rosa D, Di Campli C, Moroncini G, Manzin A, Abrignani S, Varaldo PE, Clementi M, Fadda G: Nonneutralizing human antibody fragments against hepatitis $\mathrm{C}$ virus $\mathrm{E} 2$ glycoprotein modulate neutralization of binding activity of human recombinant Fabs. Virology 2001, 288:29-35.

18. Burioni R, Plaisant P, Manzin A, Roza D, Delli Carri V, Bugli F, Solforosi L, Abrignani S, Varaldo PE, Fadda G, Clementi M: Dissection of human humoral immune response against hepatitis C virus E2 glycoprotein by repertoire cloning and generation of recombinant Fab fragments. Hepatology 1998, 28:810-814.
19. Johansson DX, Voisset C, Tarr AW, Aung M, Ball JK, Dubuisson J, Persson MA: Human combinatorial libraries yield rare antibodies that broadly neutralize hepatitis C virus. Proc Natl Acad Sci USA 2007, 104:16269-16274.

20. Kang SM, Choi SH, Park CY, Kim MH, Kim TK, Park JM, Koh MS, Kang HJ, Hwang SB: Monoclonal antibody recognizing N-terminal epitope of hepatitis C virus nonstructural 5B inhibits viral RNA replication. J Viral Hepat 2008, 15:305-313.

21. Karthe J, Tessmann K, Li J, Machida R, Daleman M, Haussinger D, Heintges $\mathrm{T}$ : Specific targeting of hepatitis $\mathrm{C}$ virus core protein by an intracellular single-chain antibody of human origin. Hepatology 2008, 48:702-712.

22. Keck ZY, Li TK, Xia J, Gal-Tanamy M, Olson O, Li SH, Patel AH, Ball JK, Lemon $S M$, Foung SK: Definition of a conserved immunodominant domain on hepatitis C virus E2 glycoprotein by neutralizing human monoclonal antibodies. J Virol 2008, 82:6061-6066.

23. Meunier JC, Russell RS, Goossens V, Priem S, Walter H, Depla E, Union A, Faulk KN, Bukh J, Emerson S, Purcell RH: Isolation and characterization of broadly neutralizing human monoclonal antibodies to the e1 glycoprotein of hepatitis C virus. J Virol 2008, 82:966-973.

24. Moradpour D, Bieck E, Hugle T, Wels W, Wu JZ, Hong Z, Blum HE, Bartenschlager R: Functional properties of a monoclonal antibody inhibiting the hepatitis $\mathrm{C}$ virus RNA-dependent RNA polymerase. J Biol Chem 2002, 277:593-601.

25. Nikonov A, Juronen E, Ustav M: Functional characterization of fingers subdomain-specific monoclonal antibodies inhibiting the hepatitis $C$ virus RNA-dependent RNA polymerase. J Biol Chem 2008, 283:24089-24102

26. Owsianka AM, Tarr AW, Keck ZY, Li TK, Witteveldt J, Adair R, Foung SK, Ball JK, Patel AH: Broadly neutralizing human monoclonal antibodies to the hepatitis C virus E2 glycoprotein. J Gen Virol 2008, 89:653-659.

27. Owsianka A, Tarr AW, Juttla VS, Lavillette D, Bartosch B, Cosset FL, Ball JK Patel AH: Monoclonal antibody AP33 defines a broadly neutralizing epitope on the hepatitis C virus E2 envelope glycoprotein. J Virol 2005, 79:11095-11104

28. Perotti M, Nancini N, Diotti RA, Tarr AW, Ball JK, Owsianka A, Adair R, Patel $\mathrm{AH}$, Clementi M, Burioni R: Identification of a broadly cross-reacting and neutralizing human monoclonal antibody directed against the hepatitis C virus E2 protein. J Virol 2008, 82:1047-1052.

29. Plaisant P, Burioni R, Manzin A, Solforosi L, Candela M, Gabrielli A, Fadda G, Clementi M: Human monoclonal recombinant Fabs specific for HCV antigens obtained by repertoire cloning in phage display combinatorial vectors. Res Virol 1997, 148:165-169.

30. Prabhu R, Khalap N, Burioni R, Clementi M, Garry RF, Dash S: Inhibition of hepatitis $C$ virus nonstructural protein, helicase activity, and viral replication by a recombinant human antibody clone. Am J Pathol 2004, 165:1163-1173.

31. Sullivan D, Mondelli M U, Curiel DT, Krasnykh V, Mikheeva G, Gaglio P, Morris CB, Dash S, Gerber MA: Construction and characterization of an intracellular single-chain human antibody to hepatitis $C$ virus nonstructural 3 protein. $J$ Hepatol 2002, 37:660-668.

32. Schofield DJ, Bartosch B, Shimizu YK, Allander T, Alter HJ Emerson SU, Cosset FL, Purcell RH: Human monoclonal antibodies that react with the E2 glycoprotein of hepatitis $C$ virus and possess neutralizing activity. Hepatology 2005, 42:1055-1062.

33. Ueno T, Misawa S, Ohba Y, Matsumoto M, Mizunuma M, Kasai N, Tsumoto $\mathrm{K}$, Kumagai I, Hayashin H: Isolation and characterization of monoclonal antibodies that inhibit hepatitis C virus NS3 protease. J Virol 2000 , 74:6300-6308

34. Yang J, Lei YF, Yin W, Wei SH, An QX, X. Lv X, Hu XB, Xu ZK: Production and characterization of monoclonal antibody specific for NS3 helicase of hepatitis C virus. Hybridoma (LarChmt) 2008, 27:181-186.

35. Biocca S, Neuberger MS, Cattaneo A: Expression and targeting of intracellular antibodies in mammalian cells. EMBO J 1990, 9:101-108.

36. Co MS, Deschamps M, Whitley RJ, Queen C: Humanized antibodies for antiviral therapy. Proc Natl Acad Sci USA 1991, 88:2869-2873.

37. Wakita T, Pietschmann T, Kato T, Date T, Miyamoto M, Zhao Z, Murthy K, Habermann A, Krausslich HG, Mizokami M, Bartenschlager R, Liang TJ: Production of infectious hepatitis $C$ virus in tissue culture from a cloned viral genome. Nat Med 2005, 11:791-796.

38. Hazari S, Chandra PK, Poat B, Datta S, Garry RF, Foster TP, Kousoulas G, Wakita T, Dash S: Impaired antiviral activity of interferon alpha against 
hepatitis C virus 2a in Huh-7 cells with a defective Jak-Stat pathway. Virology J 2010, 7:36.

39. Desogus A, Burioni R, Ingianni A, Bugli F, Pompei R, Fadda G: Production and characterization of a human recombinant monoclonal Fab fragment specific for Influenza A viruses. Clin Diagn Lab Immunol 2003, 10:680-685.

40. Gibellini D, Gardini F, Vitone F, Schiavone P, Furlini G, Carla Re M: Simultaneous detection of HCV and HIV- 1 by SYBR Green real time multiplex RT-PCR technique in plasma samples. Mol Cell Probes 2006, 20:223-229.

41. Frick DN: The hepatitis C virus NS3 protein: a model RNA helicase and potential drug target. Curr Issues Mol Biol 2007, 9:1-20.

42. Gallinari P, Brennan D, Nardi C, Brunetti M, Tomei L, Steinkuhler C, De Francesco R: Multiple enzymatic activities associated with recombinant NS3 protein of hepatitis C virus. J Virol 1998, 72:6758-6769.

43. Tai CL, Pan WC, Liaw SH, Yang UC, Hwang LH, Chen DS: Structure-based mutational analysis of the hepatitis C virus NS3 helicase. J Virol 2001, 75:8289-8297.

doi: $10.1186 / 1743-422 X-7-118$

Cite this article as: Chandra et al., Intracytoplasmic stable expression of IgG 1 antibody targeting NS3 helicase inhibits replication of highly efficient hepatitis C Virus 2a clone Virology Journal 2010, 7:118

Submit your next manuscript to BioMed Central and take full advantage of:

- Convenient online submission

- Thorough peer review

- No space constraints or color figure charges

- Immediate publication on acceptance

- Inclusion in PubMed, CAS, Scopus and Google Scholar

- Research which is freely available for redistribution

Submit your manuscript at www.biomedcentral.com/submit
C) Biomed Central 